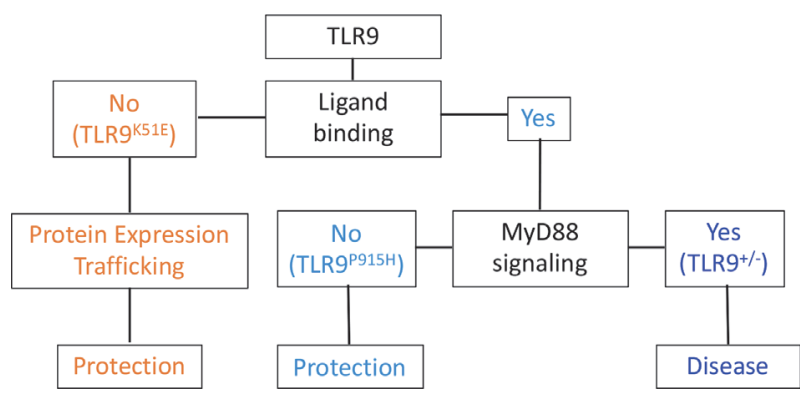

Abstract 402 Figure 1 Model explaining the implications of the TLR9 mutant disease phenotypes

the restoration of TLR9 expression induces protection. To assess the effect of TLR9-MyD88 signaling, we compared TLR9 $^{\mathrm{P} 915 \mathrm{H} / \mathrm{P9} 15 \mathrm{H}}$ and TLR9-sufficient cohorts of MRL/lpr mice. Kidney disease, survival and immune activation were significantly more severe in TLR9 ${ }^{ \pm}$mice. Thus, there is a TLR9MyD88 dependent pathway that promotes disease. Moreover, TLR9 ${ }^{\mathrm{K} 51 \mathrm{E} /-}$ mice had increased glomerulonephritis and immune activation compared to TLR9 ${ }^{\mathrm{P} 915 \mathrm{H} / \mathrm{P} 915 \mathrm{H}}$ mice, suggesting that TLR9 could regulate disease through a ligand binding-dependent but MyD88-independent mechanism (figure 1).

Using a 3 way (TLR9 ${ }^{\mathrm{WT}}$, TLR9 ${ }^{\mathrm{P} 915 \mathrm{H}}$ and TLR9 $^{--}$)-mixed bone marrow chimera, we found that TLR9 inhibits B cell development and differentiation in a B cell-intrinsic fashion and that the absence of TLR9 $\left(\mathrm{TLR}^{-/}\right.$) was very different from the inability of TLR9 to signal (TLR9 $\left.{ }^{\mathrm{P} 915 \mathrm{H}}\right)$. RNA seq analysis of sorted age-associated $\mathrm{B}$ cells $(\mathrm{ABC})$ revealed that TLR9 ${ }^{\mathrm{WT}}$, TLR9 $^{\mathrm{P} 915 \mathrm{H}}$ and $\mathrm{TLR}^{-/-} \mathrm{ABC}$ exhibit different transcriptional programs. Notably, the absence of TLR9 did not lead to an increase in genes that are induced by TLR7, arguing against the idea that TLR9 simply restrains TLR7 signaling.

Conclusion This in vivo genetic dissection of TLR9 reveals how it both promotes and regulates lupus. Inability of TLR9 to signal via MyD88 is different from absence of TLR9 and also is different from inability of TLR9 to bind ligand. These findings shed light on the basic biology of endosomal TLR signaling and are relevant to the design of TLR-targeted therapy. Acknowledgement Funded by R37-AI118841 (M. Shlomchik).

\section{BACTERIAL BIOFILM PRODUCT CURLI/EDNA INDUCES NEUTROPHIL EXTRACELLULAR TRAPS AND SERUM ANTI-CURLI/EDNA LEVELS CORRELATE WITH BACTERIURIA AND LUPUS ACTIVITY}

${ }^{1}$ Ryan J Pachucki, ${ }^{1}$ Lynne Kohler, ${ }^{2}$ Stefania Gallucci, ${ }^{2}$ Çagla Tükel, ${ }^{1}$ Roberto Caricchio* ${ }^{1}$ Division of Rheumatology; ${ }^{2}$ Department of Microbiology, Lewis Katz School of Medicine, Temple University, Philadelphia, PA

\subsection{6/lupus-2021-lupus21 century. 16}

Background Infections are a major contributor to lupus disease. We have previously demonstrated that bacterial amyloid curli, produced by E.coli, can accelerate disease in mouse models of lupus. Interestingly curli incorporates extracellular DNA, which in turn can be both adjuvant and a self-antigen in lupus. Uropathogenic E. coli (UPEC) is responsible for the majority of urinary tract infections in SLE. Based on our previous results, we hypothesize that exposure to UPEC triggers anti-curli/eDNA antibodies and curli/eDNA complexes can trigger the innate immune system.

Methods We investigated 98 lupus patients who met at least 4 SLICC criteria. Results were compared to 54 age, sex and race matched healthy controls. We tested the production of anti-curli/DNA complex for both $\operatorname{IgG}$ and $\operatorname{IgA}$ subclasses. We than correlated the levels of anti-curli/DNA antibodies with clinical parameters. Finally, we treated human neutrophils with curli/eDNA complexes.

Results We found that curli/eDNA induces neutrophil extracellular traps in a ROS-dependent manner. Anti-curli/eDNA IgG levels were detected in lupus and controls plasma and the levels correlated with persistent bacteriuria $(p<0.05)$ and disease flares in lupus patients. In addition, anti-dsDNA could bind to anti-curli/eDNA complexes.

Conclusions We conclude curli/eDNA complexes can activate the innate and adaptive immune system and could be a mechanism to sustain disease in lupus.

Acknowledgments We thank Drs. Marc Monestier and Philip Cohen for their insightful suggestions. We also thank the generosity of the lupus patients from the Temple Lupus Program.

\section{PLATELETS ARE A SOURCE OF EXTRACELLULAR MITOCHONDRIA AND MITOCHONDRIAL DNA IN SYSTEMIC LUPUS ERYTHEMATOSUS}

\begin{abstract}
1,2Imene Melki, 1,2 Isabelle Allaeys, 1,2Nicolas Tessandier, 1,2Tania Lévesque, ${ }^{1}$ Nathalie Cloutier, ${ }^{1,2}$ Audrée Laroche, ${ }^{3}$ Nathalie Vernoux, ${ }^{1,2}$ Yann Becker, ${ }^{1,2}$ Hadrien BenkFortin, ${ }^{1,2}$ Anne Zufferey, ${ }^{1,2}$ Emmanuelle Rollet-Labelle, ${ }^{1,2}$ Marc Pouliot, ${ }^{4}$ Guy Poirier, ${ }^{5}$ Natacha Patey, ${ }_{6}^{6}$ Clemence Belleannee, ${ }^{4}$ Denis Soulet, ${ }^{7}$ Steven E McKenzie, ${ }^{8}$ Alain Brisson, ${ }^{3,9}$ Marie-Eve Tremblay, ${ }^{10}$ Christian Lood, ${ }^{1,2,11}$ Paul R Fortin*, Eric Boilard ${ }^{* 1,2} .{ }^{1}$ Centre de recherche du Centre hospitalier universitaire de Québec-Université Laval, Québec, QC, GIV 4G2, Canada; ${ }^{2}$ Faculté de Médecine and Centre de recherche ARThrite, Université Laval, Québec, QC, GIV 4G2, Canada; ${ }^{3}$ Axe neurosciences du Centre de recherche du Centre hospitalier universitaire de Québec-Université Laval et Département de médecine moléculaire de I'Université Laval, Québec, QC, GIV 4G2, Canada; ${ }^{4}$ Department of Molecular Biology, Medical Biochemistry, and Pathology, Faculty of Medicine, Université Laval, Quebec, QC, GIV 4G2, Canada; ${ }^{5}$ Centre Hospitalier Universitaire de Sainte-Justine, Faculté de Médecine, Département de pathologie et biologie cellulaire, Université de Montréal, Montréal, QC, H3T 1C5, Canada; ${ }^{6}$ Department of Obstetrics, Gynecology and Reproduction, Centre hospitalier universitaire (CHU) de Québec-Université Laval et Département de médecine moléculaire de I'Université Laval, Québec, QC, GIV 4G2, Canada; ${ }^{7}$ Cardeza Foundation for Hematologic Research, Thomas Jefferson University, Philadelphia, PA 19107, USA; ${ }^{8}$ UMR-CBMN CNRS-Université de Bordeaux-IPB, Pessac 33600, France; ${ }^{9}$ Division of Medical Sciences, University of Victoria, Victoria, BC, V8W 2Y2, Canada; ${ }^{10}$ Division of Rheumatology, Department of Medicine, University of Washington, Seattle, WA 98109, USA; "1D Division of Rheumatology, Department of Medicine, CHU de Québec-Université Laval, Québec, QC, GIV 4G2, Canada
\end{abstract}

\subsection{6/lupus-2021-lupus21 century. 17}

Background The accumulation of DNA and nuclear components in blood and their recognition by autoantibodies play a central role in the pathophysiology of systemic lupus erythematosus (SLE). Despite the efforts, the sources of these circulating autoantigens in SLE are still unclear. While damaged organs and activated cells are generally considered as potential sources of autoantigens, platelets are often overlooked given that they are anucleated and thereby cannot release genomic DNA. However, accumulating findings suggest that mitochondria are also targeted by antibodies in SLE.

Methods We examined the presence of extracellular mitochondria in blood of patients with SLE and determined correlations with platelet activation. Because mice lack Fc $\gamma$ RIIA and murine platelets are completely devoid of receptor capable of binding IgG-containing immune complexes, we generated transgenic lupus mice expressing FcyRIIA for our in vivo investigations. We used a reporter mouse with red fluorescent protein targeted to the mitochondrion to identify the cellular source of the extracellular mitochondria. 